\title{
Effects of Explanatory Variables in Count Data Moving Average Models
}

\author{
Kurt Brännäs and Carl Lönnbark* \\ Department of Economics, Umeå University \\ SE-901 87 Umeå, Sweden
}

\begin{abstract}
This note gives dynamic effects of discrete and continuous explanatory variables for count data or integer-valued moving average models. An illustration based on a model for the number of transactions in a stock is included.
\end{abstract}

JEL Classification: C22, C25, G12.

Key Words: INMA model, Marginal effect, Intra-day, Financial data.

Umeå Economic Studies 679, 2006

\section{Introduction}

This note gives dynamic effects of explanatory variables for count data or integer-valued moving average (INMA) models with time dependent parameters.

The INMA model of order $q$ was introduced by Al-Osh and Alzaid (1988) and McKenzie (1988). Brännäs and Hall (2001) summarize previous model characterizations and add two new ones. In essence, the model can be interpreted in alternative ways, but importantly the first both conditional and unconditional moments remain the same. Higher order moments such as autocorrelations differ for the different interpretations.

When it comes to introducing explanatory variables our focus is on the first order moment functions, and obviously we wish to adhere to an integervalued data generating process. This can be accomplished by letting parameters become functions of explanatory variables and by adopting functional forms that are consistent with an INMA process. Brännäs et al. (2002) and Brännäs

\footnotetext{
*The financial support to the former author from the Wallander-Hedelius Foundation is gratefully acknowledged.
} 
and Quoreshi (2006) give empirical results for INMA model with explanatory variables in tourism and financial research settings. Given such specifications, obtaining marginal and other dynamic effects on the conditional mean over time is feasible and of great practical interest.

\section{Model}

$\operatorname{An} \operatorname{INMA}(q)$ can be written as

$$
y_{t}=\theta_{0, t} \circ \varepsilon_{t}+\theta_{1, t-1} \circ \varepsilon_{t-1}+\theta_{2, t-2} \circ \varepsilon_{t-2}+\ldots+\theta_{q, t-q} \circ \varepsilon_{t-q},
$$

where $\circ$ indicates binomial thinning and the INMA parameters, $\theta_{0, t}=1, \theta_{i, t} \in$ $[0,1], i=1, \ldots, q-1$, and $\theta_{q, t} \in(0,1]$, are time dependent thinning probabilities. ${ }^{1}$ In addition, $E\left(\varepsilon_{t}\right)=\lambda_{t}$. This representation is a generalized model in that both the $\theta_{i, t} \mathrm{~s}$ and the $\lambda_{t}$ are time dependent. It is through these parameters that we most simply can include exogenously determined explanatory variables contained in a vector $\mathbf{x}_{t}$.

For INAR models it appears reasonable to include the effect of explanatory variables through the AR-parameters, and possibly in terms of $\lambda_{t}$ (Brännäs, 1995). When it comes to including explanatory in INMA models, Brännäs et al. (2002) in an INMA(1) model lets both $\theta_{1, t}$ and $\lambda_{t}$ vary, while Brännäs and Quoreshi (2006) lets only $\lambda_{t}$ vary with explanatory variables.

For the $\operatorname{INMA}(q)$ and conditional on $\mathbf{x}_{t}$ it holds that

$$
E\left(y_{t}\right)=\theta_{0, t} \lambda_{t}+\theta_{1, t-1} \lambda_{t-1}+\theta_{2, t-2} \lambda_{t-2}+\ldots+\theta_{q, t-q} \lambda_{t-q} .
$$

For $\theta_{i, t}$ a logistic specification is chosen, i.e. $\theta_{i, t}=1 /\left[1+\exp \left(\mathbf{x}_{t} \boldsymbol{\beta}_{i}\right)\right]$, while for $\lambda_{t}$ we consider a static as well as a dynamic specification. Obviously, other specifications are also possible.

\section{Dynamic Effects}

\subsection{Time Dependent $\lambda_{t}$}

Consider first the conventional count data specification $\lambda_{t}=\exp \left(\mathbf{x}_{t} \boldsymbol{\beta}\right)$ together with time invariant $\theta_{i}, i=1, \ldots, q$. Then $\partial \lambda_{t} / \partial x_{k, t}=\beta_{k} \lambda_{t}$, with $k$ indicating the continuous $x$-variable of interest. Since, $\partial E\left(y_{t}\right) / \partial \lambda_{t-s}=\theta_{s}$, for $s \leq q$, and equal to zero otherwise, a marginal change in variable $k$ at time $t-s$ gives the marginal effect

$$
m_{t, s}^{k}=\frac{\partial E\left(y_{t}\right)}{\partial x_{k, t-s}}=\frac{\partial E\left(y_{t}\right)}{\partial \lambda_{t-s}} \frac{\partial \lambda_{t-s}}{\partial x_{k, t-s}}=\left\{\begin{array}{ll}
\theta_{s} \beta_{k} \lambda_{t-s}, & s \leq q \\
0, & s>q
\end{array} .\right.
$$

\footnotetext{
${ }^{1}$ The binomial thinning operator $\alpha \circ x=\sum_{i=1}^{x} z_{i}$, where $\left\{z_{i}\right\}$ is a $0-1$ iid random sequence, with $\operatorname{Pr}\left(z_{i}=1\right)=\alpha$ as the thinning probability. Conditionally on $x, \alpha \circ x$ is binomially distributed with mean $\alpha x$ and variance $\alpha(1-\alpha) x$. Given independence between the $z_{i}$ and $x, E(\alpha \circ x)=\alpha E(x)$ and $V(\alpha \circ x)=\alpha^{2} V(x)+\alpha(1-\alpha) E(x)$. Also, $\alpha \circ x \in[0, x]$.
} 
We may determine the variance for the effect by the delta method, i.e. $V\left(m_{k, s}\right)=$ $V\left(\hat{\theta}_{s} \hat{\beta}_{k} \hat{\lambda}_{t-s}\right) \approx \mathbf{h}^{\prime} V(\boldsymbol{\psi}) \mathbf{h}$, where $\boldsymbol{\psi}^{\prime}=\left(\theta_{1}, \ldots, \theta_{q} ; \beta_{1}, \ldots, \beta_{m}\right)$, and both the covariance matrix of the parameter estimator, i.e. $V(\boldsymbol{\psi})$, and $\mathbf{h}=\partial m_{k, s} / \partial \boldsymbol{\psi}$ are evaluated at the estimates.

If $x_{k}$ is a discrete-valued variable, say a dummy variable, it appears more reasonable to give results of a unit change in $x_{k}$ in terms of absolute or relative changes in the mean. Let $\mathbf{x}_{t}$ denote the base level and the new level by $\mathbf{x}_{t}^{k}$ for a unit change in variable $k$. Then

$$
\begin{aligned}
\nabla_{t, s}^{k} & =E\left(y_{t} \mid \mathbf{x}_{t-s}^{k}\right)-E\left(y_{t} \mid \mathbf{x}_{t-s}\right)=\theta_{s} \exp \left(\mathbf{x}_{t-s} \boldsymbol{\beta}\right)\left[\exp \left(\beta_{k}\right)-1\right] \\
& \approx \theta_{s} \beta_{k} \lambda_{t-s}
\end{aligned}
$$

and the percentage change

$$
\begin{aligned}
D_{t, s}^{k} & =100 \frac{E\left(y_{t} \mid \mathbf{x}_{t-s}^{k}\right)-E\left(y_{t} \mid \mathbf{x}_{t-s}\right)}{E\left(y_{t} \mid \mathbf{x}_{t-s}\right)} \\
& =100\left(e^{\beta_{k}}-1\right)\left(\theta_{s} \lambda_{t-s} / \sum_{i=0}^{q} \theta_{i} \lambda_{t-i}\right) \approx 100 \beta_{k}\left(\theta_{s} \lambda_{t-s} / \sum_{i=0}^{q} \theta_{i} \lambda_{t-i}\right) .
\end{aligned}
$$

The approximations are for small $\beta_{k}$.

Consider next a case of a dynamic specification $\lambda_{t}=\lambda_{t-1}^{\alpha} \exp \left(\mathbf{x}_{t} \boldsymbol{\beta}\right)$. Since $\ln \lambda_{t}=\alpha \ln \lambda_{t-1}+\mathbf{x}_{t} \boldsymbol{\beta}$ and since $\partial \ln \lambda_{t} / \partial x_{k, t-s}=\left(\partial \ln \lambda_{t} / \partial \lambda_{t}\right)\left(\partial \lambda_{t} / \partial x_{k, t-s}\right)$ it follows that $\partial \lambda_{t} / \partial x_{k, t-s}=\lambda_{t}\left(\partial \ln \lambda_{t} / \partial x_{k, t-s}\right)$. By recursive substitution

$$
\ln \lambda_{t}=\alpha^{t} \ln \lambda_{0}+\sum_{i=1}^{t} \alpha^{t-i} \mathbf{x}_{i} \boldsymbol{\beta}
$$

Therefore, $\partial \ln \lambda_{t} / \partial x_{k, t-s}=\alpha^{s} \beta_{k}$ and $\partial \lambda_{t} / \partial x_{k, t-s}=\alpha^{s} \beta_{k} \lambda_{t}$. In the $\operatorname{INMA}(q)$ model we then get the marginal effect

$$
m_{t, s}^{k}=\sum_{i=0}^{s} \theta_{i} \frac{\partial \lambda_{t-i}}{\partial x_{k, t-s}}=\beta_{k} \sum_{i=0}^{s} \alpha^{s-i} \theta_{i} \lambda_{t-i}
$$

with here and in the sequel $\theta_{0}=1$ and $\theta_{i}=0, i>q$. The effects of a discrete change of a unit size in a variable $x_{k}$ are given by

$$
\begin{aligned}
\nabla_{t, s}^{k} & =\sum_{i=0}^{s} \theta_{i} \lambda_{t-i}\left[\exp \left(\beta_{k} \alpha^{s-i}\right)-1\right] \\
D_{t, s}^{k} & =100 \nabla_{t, s}^{k} / \sum_{i=0}^{q} \theta_{i} \lambda_{t-i} .
\end{aligned}
$$

For the more general case of

$$
\lambda_{t}=\lambda_{t-1}^{\alpha_{1}} \ldots \lambda_{t-p}^{\alpha_{p}} \exp \left(\mathbf{x}_{t} \boldsymbol{\beta}\right)
$$


we employ the companion form

$$
\left(\begin{array}{c}
\ln \lambda_{t} \\
\ln \lambda_{t-1} \\
\vdots \\
\ln \lambda_{t-p+1}
\end{array}\right)=\left(\begin{array}{cccc}
\alpha_{1} & \alpha_{2} & \ldots & \alpha_{p} \\
1 & 0 & \ldots & 0 \\
\vdots & \ddots & \ddots & \vdots \\
0 & \ldots & 1 & 0
\end{array}\right)\left(\begin{array}{c}
\ln \lambda_{t-1} \\
\ln \lambda_{t-2} \\
\vdots \\
\ln \lambda_{t-p}
\end{array}\right)+\left(\begin{array}{c}
\mathbf{x}_{t} \boldsymbol{\beta} \\
0 \\
\vdots \\
0
\end{array}\right)
$$

or

$$
\boldsymbol{\Lambda}_{t}=\mathbf{A} \boldsymbol{\Lambda}_{t-1}+\mathbf{z}_{t}=\mathbf{A}^{t} \boldsymbol{\Lambda}_{0}+\sum_{i=1}^{t} \mathbf{A}^{t-i} \mathbf{z}_{i}
$$

We assume that the largest eigenvalue of $\mathbf{A}$ is smaller than one, so that $\mathbf{A}^{t} \rightarrow 0$ with increasing $t$. Here, $t$ is assumed large. Since $\partial \mathbf{z}_{i} / \partial x_{k, i}=\left(\beta_{k}, 0, \ldots, 0\right)^{\prime}$ and $\mathbf{A}^{k}=\mathbf{R} \boldsymbol{\theta}^{k} \mathbf{S}$, with $\mathbf{R}$ the right and $\mathbf{S}$ the left matrix of eigenvectors, respectively, and $\Theta$ the diagonal matrix of eigenvalues, we have

$$
\frac{\partial \ln \lambda_{t}}{\partial x_{k, t-s}}=\mathbf{1}^{\prime} \frac{\partial \boldsymbol{\Lambda}_{t}}{\partial x_{k, t-s}}=\beta_{k} \mathbf{1}^{\prime} \mathbf{R} \boldsymbol{\theta}^{s} \mathbf{S} \mathbf{1}
$$

where $\mathbf{1}=(1,0, \ldots, 0)^{\prime}$. We use this result and $\partial \lambda_{t} / \partial x_{k, t-s}=\lambda_{t} \partial \ln \lambda_{t} / \partial x_{k, t-s}$ to obtain

$$
m_{t, s}^{k}=\beta_{k} \sum_{i=0}^{s} \theta_{i} \lambda_{t-i} \mathbf{1}^{\prime} \mathbf{R} \boldsymbol{\theta}^{s-i} \mathbf{S} \mathbf{1} .
$$

The effects of a discrete, unit change in variable $x_{k}$ are in this case given by

$$
\begin{aligned}
\nabla_{t, s}^{k} & =\sum_{i=0}^{s} \lambda_{t-i} \theta_{i}\left[\exp \left(\beta_{k} \mathbf{1}^{\prime} \mathbf{A}^{s-i} \mathbf{1}\right)-1\right] \\
D_{t, s}^{k} & =100 \frac{\sum_{i=0}^{s} \lambda_{t-i} \theta_{i}\left[\exp \left(\beta_{k} \mathbf{1}^{\prime} \mathbf{A}^{s-i} \mathbf{1}\right)-1\right]}{\sum_{i=0}^{q} \theta_{i} \lambda_{t-i}} .
\end{aligned}
$$

\subsection{Time Dependent $\theta_{i, t}$}

In this case $\lambda_{t}=\lambda$ and $\partial \theta_{i, t} / \partial x_{k, t}=-\beta_{i, k} \theta_{i, t}\left(1-\theta_{i, t}\right)$, for $s \leq q$, so that

$$
m_{t, s}^{k}=-\lambda \beta_{s, k} \theta_{i, t-s}\left(1-\theta_{s, t-s}\right) .
$$

The effects of a discrete change in $x_{k}$ are

$$
\begin{aligned}
& \nabla_{t, s}^{k}=\lambda\left(\theta_{i, s}^{k}-\theta_{i, s}\right) \\
& D_{t, s}^{k}=100\left(\theta_{i, s}^{k}-\theta_{i, s}\right) / \sum_{i=0}^{q} \theta_{i, t-i},
\end{aligned}
$$

where superscript $k$ denotes the new level. 


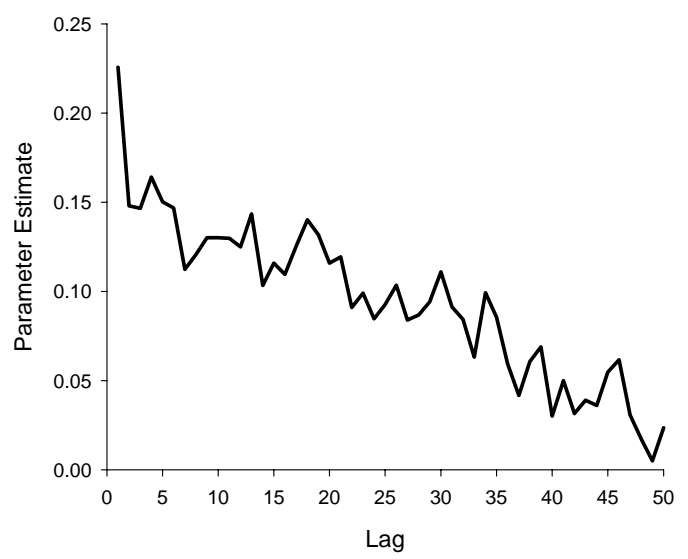

Figure 1: Estimates of moving average parameters.

\subsection{Time Dependent $\theta_{i, t}$ and $\lambda_{t}$}

Setting $\theta_{i, t}=1 /\left[1+\exp \left(\mathbf{x}_{t} \boldsymbol{\beta}_{i}\right)\right]$ and $\lambda_{t}=\exp \left(\mathbf{w}_{t} \boldsymbol{\gamma}\right)$ and letting $x_{k, t}=w_{l, t}$ be the variables of which we wish to obtain effects we get the marginal effect on the form

$$
m_{t, s}^{k l}=\theta_{s, t-s} \lambda_{t-s}\left[\gamma_{l}-\beta_{s, k}\left(1-\theta_{s, t-s}\right)\right]
$$

and

$$
\begin{aligned}
\nabla_{t, s}^{k l} & =\lambda_{t-s}\left[e^{\beta_{k}} \theta_{s, t-s}^{l}-\theta_{s, t-s}\right] \\
D_{t, s}^{k l} & =100 \nabla_{t, s}^{k l} / \sum_{i=0}^{q} \theta_{i, t} \lambda_{t-i} .
\end{aligned}
$$

\section{Empirical Illustration}

The empirical illustration is based on the INMA(50) model for intra-day data of Brännäs and Quoreshi (2006). The model explains the number of traded stocks in Ericsson B, with 50 INMA-lags, see Figure 1 for the estimates, and a time dependent $\lambda_{t}$ function. The $\lambda_{t}$ is of the form $\lambda_{t-1}^{\alpha} \exp \left(\mathbf{x}_{t} \boldsymbol{\beta}\right)=\exp (0.283+$ $\left.0.328 \ln \lambda_{t-1}-3.892 \nabla p_{t}-4.154 \nabla p_{t}^{+}+11.128 \nabla s_{t}+0.242 \cdot 1_{t}\right)$, where $\nabla p_{t}^{+}=0$ for $\nabla p_{t} \leq 0$ and $\nabla p_{t}^{+}=\nabla p_{t}$ for positive price changes, $\nabla s_{t}$ is a spread change, and $1_{t}=1(t \leq 1100)$. The explanatory variables are discrete and we set $\lambda_{0}=8$, $\nabla s_{t}=0$ and $1_{t}=1$.

Figure 2 reports effects $\left(\nabla_{t, s}^{k}\right)$ over time of price changes at time $t=50$. To calculate the effects we use an extension of the $\nabla_{t, s}^{k}$ of Section 3.1, i.e. $\nabla_{t, s}^{k}=\sum_{i=0}^{s} \theta_{i} \lambda_{t-i}\left[\exp \left(\alpha^{s-i} \delta_{k s} \beta_{k}\right)-1\right]$, with $\delta_{k s}= \pm 2$ and \pm 5 . Two features 


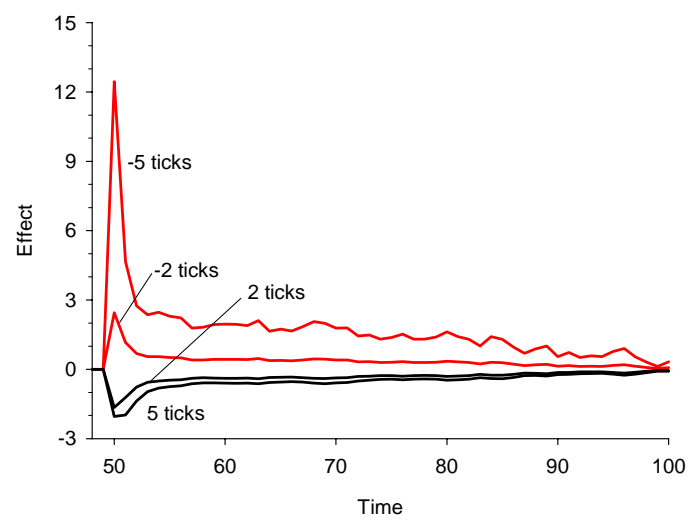

Figure 2: Effects of discrete price changes at $t=50$.

are noteworthy. First, effects converge the further we move from the period of change. Smaller changes gives faster convergence. Second, the effects of positive and negative changes in price are not symmetrical. The initial effect of a negative change increases trading frequency by much more than the trade frequency decrease due to a positive change. Over all lags the effects of a -5-ticks change is about 2.5 times larger, while there is hardly any size difference for the \pm 2 -ticks changes.

\section{References}

Al-Osh M. and Alzaid A. (1988). Integer-Valued Moving Average (INMA) Process. Statistical Papers 29, 281-300.

Brännäs, K. (1995). Explanatory Variables in the AR(1) Count Data Model. Umeå Economic Studies 381.

Brännäs, K. and Hall, A. (2001). Estimation in Integer-Valued Moving Average Models. Applied Stochastic Models in Business and Industry 17, 277-291.

Brännäs, K., Hellström, J. and Nordström, J. (2002). A New Approach to Modelling and Forecasting Monthly Guest Nights in Hotels. International Journal of Forecasting 18, 19-30.

Brännäs, K. and Quoreshi, A.M.M.S. (2006). Integer-Valued Moving Average Modelling of the Number of Transactions in Stocks. Umeå Economic Studies 637.

McKenzie, E. (1988). Some ARMA models for Dependent Sequences of Poisson Counts. Advances in Applied Probability 20, 822-835. 